\title{
An Exploratory Study of The Perceptions of Footwear for Individuals who use Lower Limb Orthotics
}

\author{
Margaret E. Gegen \\ Belmont University, megegen@gmail.com \\ Teresa Plummer \\ Belmont University, teresa.plummer@belmont.edu \\ Nancy Darr \\ Belmont University, nancy.darr@belmont.edu
}

Follow this and additional works at: https://nsuworks.nova.edu/ijahsp

Part of the Accessibility Commons, Child Psychology Commons, Developmental Psychology Commons, Occupational Therapy Commons, Orthotics and Prosthetics Commons, Physical Therapy Commons, and the Social Psychology Commons

\section{Recommended Citation}

Gegen ME, Plummer T, Darr N. An Exploratory Study of The Perceptions of Footwear for Individuals who use Lower Limb Orthotics. The Internet Journal of Allied Health Sciences and Practice. 2020 Jan 01;18(4), Article 8.

This Manuscript is brought to you for free and open access by the College of Health Care Sciences at NSUWorks. It has been accepted for inclusion in Internet Journal of Allied Health Sciences and Practice by an authorized editor of NSUWorks. For more information, please contact nsuworks@nova.edu. 


\title{
An Exploratory Study of The Perceptions of Footwear for Individuals who use Lower Limb Orthotics
}

\begin{abstract}
The purpose of this study was to explore individual's perceptions of orthotics and footwear. A survey was created by the authors with the expert opinions of physical and occupational therapists and was distributed to two therapy clinics in Tennessee that provide both occupational therapy and physical therapy as well as on several online support groups. Seventy-nine (79) caregivers completed surveys. Thematic analysis was performed using Nvivo 10 (QSR International) and descriptive statistics were generated using the Statistical Package for the Social Sciences (SPSS) IBM International). Ninety percent (90\%) of participants report that orthotics affect what type of shoes the user can wear. Eighty-two percent (82\%) report that it is difficult to shop for shoes that accommodate orthotics. Sixty-one percent (61\%) report that the user would be more likely to wear their orthotics if they had more options of shoes to wear. Fifty-nine percent $(59 \%)$ report that the user would be more likely to wear their orthotics if they had shoes that were more attractive. The importance of collaborating with consumers when designing products is essential for the success of both the individual and the product.
\end{abstract}

\section{Author Bio(s)}

- Margaret Gegen, OTD, OTR/L, is an occupational therapist in Nashville, TN.

- Dr. Nancy Darr, PT, DSc, NCS is a professor in the physical therapy department at Belmont University and a pediatric therapist in Nashville, TN. She is certified as a clinical specialist in Neurologic Physical Therapy by the American Board of Physical Therapy Specialties.

- Dr. Teresa Plummer, PHD, OTR/L, ATP, CAPS, CEAS is a professor in the occupational therapy department at Belmont University. She Dr. Plummer serves on the Editorial Board for Rehab Management and is a reviewer for the American Journal of Occupational Therapy. She has co-authored the RESNA position paper on Pediatric Power Mobility (2009). 


\title{
IIIAHSP \\ The Internet Journal of Allied Health Sciences and Practice
}

Dedicated to allied health professional practice and education

Vol. 18 No. 4 ISSN 1540-580X

\section{An Exploratory Study of The Perceptions of Footwear for Individuals who use Lower Limb Orthotics}

\author{
Margaret E. Gegen \\ Teresa Plummer \\ Nancy Darr \\ Belmont University \\ United States
}

\begin{abstract}
The purpose of this study was to explore individual's perceptions of orthotics and footwear. A survey was created by the authors with the expert opinions of physical and occupational therapists and was distributed to two therapy clinics in Tennessee that provide both occupational therapy and physical therapy as well as on several online support groups. Seventy-nine (79) caregivers completed surveys. Thematic analysis was performed using Nvivo 10 (QSR International) and descriptive statistics were generated using the Statistical Package for the Social Sciences (SPSS) IBM International). Ninety percent (90\%) of participants report that orthotics affect what type of shoes the user can wear. Eighty-two percent (82\%) report that it is difficult to shop for shoes that accommodate orthotics. Sixty-one percent $(61 \%)$ report that the user would be more likely to wear their orthotics if they had more options of shoes to wear. Fifty-nine percent (59\%) report that the user would be more likely to wear their orthotics if they had shoes that were more attractive. The importance of collaborating with consumers when designing products is essential for the success of both the individual and the product.
\end{abstract}

Keywords: satisfaction, lower limb orthotics, AFO, footwear, perception 


\section{INTRODUCTION}

According to The U.S. Census Bureau, $6.9 \%(20,903,105)$ of Americans have difficulties with ambulation/mobility. Of these individuals, $0.6 \%(324,125)$ are children under the age of $18 .{ }^{1}$ In order to compensate for these mobility deficits, individuals often utilize assistive technology (AT). AT is defined as any item or piece of equipment that is used to increase, maintain, or improve the functional capabilities of individuals with disabilities. ${ }^{2}$ A lower limb orthotics is a piece of AT or an assistive device provided to individuals with mobility impairments to improve gait, prevent deformities, promote engagement in important occupations, and to overcome difficulties completing functional activities. Many AT devices are often abandoned shortly after they are prescribed. "Disuse of AT is understood to be due to a host of reasons, ranging from an individual feeling uncomfortable or embarrassed with their AT, to an individual finding the device too heavy or awkward to use."3 This is problematic and can have profound effects on both the physical health and the quality of life of individuals who benefit from the use of AT. The same goes for the use of shoes for individuals who require special orthopedic considerations or who wear orthotics that do not fit into traditional shoes. "Finding the right AT to meet an individual's needs may greatly reduce the disuse of AT and consequently, better support people with disabilities." ${ }^{3}$

For some individuals with disabilities, they are unable to access their environment and fully participate in daily activities without these devices. Research suggests that individuals who engage in leisure activities have better psychological and physical functioning as well as higher life satisfaction, and lower depression. ${ }^{4}$ Not only is it important to support individual's physical health through providing the optimal AT, but it is also important to support their mental health. While research in this field is limited, current research suggests that footwear is strongly connected to an individual's body image and self-esteem and thus, their mood, depression, and quality of life in individuals with Rheumatoid Arthritis. ${ }^{5}$ In addition, research conducted by Polliack, Elliot, Caves, McNeal, and Landsberger suggests that footwear affects the design of the orthotic, compliance with the orthotic, and self-esteem. ${ }^{6}$ With this information, it becomes apparent that it is important to take into consideration the needs, wants, and considerations of the clients and thus, further investigate patient perceptions of footwear and incorporate client choice into the design process.

The perceptions of individuals with disabilities are often neglected throughout the design phase of creating footwear. In order to increase satisfaction and use of both orthotics and footwear, it is important to first gain the perceptions of individuals who use lower limb orthotics and their caregivers regarding orthotics and footwear. By understanding the footwear needs of individuals who use lower limb orthotics, designers and manufacturers may be able to create footwear that meets both the personal needs of the population (i.e. color, weight, appearance etc.) as well as allow the orthotic to function as medically necessary. Designing products with a client-centered approach will increase quality of life, improve patient outcomes, and decrease the abandonment of products. This study explores the perceptions that caregivers (of individuals who use lower limb orthotics) have regarding orthotics and footwear.

\section{METHODS}

Caregivers of individuals who wear lower limb orthotics were recruited from two outpatient clinics (Franklin, TN and Clarksville, TN) that provide occupational therapy (OT), physical therapy (PT), and speech therapy (ST) for individuals ages 0-26 years old. Many individuals who receive therapy at these clinics wear orthotics to compensate for hypotonicity, weakness, or anatomical structure. At both clinics, an orthotist works closely with the therapists and the families to provide orthotics that meet the medical needs of the user. Some diagnoses that are commonly treated at these clinics include cerebral palsy, Down syndrome, and Rett syndrome. Individuals who receive therapy are often working on gaining strength, developing gross motor skills, and refining fine motor skills through both occupational and physical therapy to further promote function and independence. Physical therapists and occupational therapists at the two clinics were educated on the inclusion criteria for the study and invited caregivers who met the criteria to participate in the study. The caregivers were required to be at least 18 years old and have a child or care for a child who utilized lower limb orthotics. For individuals who were recruited from Facebook groups, an invitation was posted stating the inclusion criteria. Individuals who met the inclusion criteria were provided a link to the survey. The Facebook groups that were utilized were for caregivers of individuals who utilized lower limb orthotics, whose children had experienced cerebral palsy, Rett's syndrome, Down syndrome, clubfoot, Charcot Marie tooth disease, or a cerebrovascular accident resulting in the recommended use of an orthotic. The individuals who participated were required to cognitively be able to understand and answer questions in English. All participants received information prior to completing the questionnaire to obtain consent. This study was approved by the Belmont University Institutional Review Board.

The survey was developed by 6 clinicians that work with individuals who utilize lower limb orthotics. The survey was inspired by reviewing literature as well as by discovering in clinical practice that a limited number of products were available to the population. Questions were created with the expert opinions of physical therapists and occupational therapists who were able to provide relevant information relating to the use of orthotics and footwear. Nancy Darr, PT, DSc, NCS, contributed to the creation of the survey. She has a wealth of experience (practicing since 1985) and has extensive experience in creating orthotics for children. In 
current practice she frequently collaborates with orthotists to ensure the orthotic is the best fit for the individual. She is a Boardcertified specialist in Neurologic physical therapy and has extensive experience conducting quantitative research. Another physical therapist who contributed to the creation of survey questions, Ellen Argo, PT, PCS, C/NDT, has been a practicing physical therapist since 2000 and has extensive experience in the area of orthotics and research specifically with the pediatric population. The third individual who contributed to the surveys is Teresa Plummer, PhD, MSOT, OTR/L, CAPS, CEAS, ATP, an occupational therapist who has over 35 years of experience in the field and is an expert in the field of qualitative research. The survey consisted of 25 questions and included both open and closed ended questions to gather both qualitative and quantitative data. The survey has not been validated. To ensure that questions were not leading, the researchers/authors carefully worded the questions in a neutral manner as to not assume a response or to make the participant feel like they were right or wrong for answering a certain way.

The survey was distributed to each clinic and therapists at each facility were provided with packets to distribute to caregivers who met the inclusion criteria. Each packet included a letter of invitation explaining the study purpose, procedures, and participation criteria and a caregiver survey. Therapists then asked caregivers who met the inclusion criteria to complete the survey and return to designated personnel in the sealed envelope provided with each study packet. By returning the survey, consent was implied. Participants were also recruited using Facebook support groups. Participants that were recruited via Facebook provided responses via a Qualtrics survey. For all participants, any person who met the inclusion criteria was invited to answer the survey. The therapists at the clinics did not individually seek out families but rather invited all who qualified. No identifying information was collected from any participants. Data was analyzed based on all answered responses and did not account for unanswered questions.

\section{Data Analysis}

The study design was a mixed method concurrent nested design with both qualitative and quantitative data being obtained from the surveys. ${ }^{6}$ Qualitative data from open-ended questions was analyzed through thematic analysis using Nvivo 10 (QSR International). With an inductive qualitative approach, common themes were identified based on word-frequency statistics. Three researchers completed the thematic analysis to ensure that the common themes chosen were mutually agreed upon and unbiased. Descriptive statistics were generated using The Statistical Package for the Social Sciences ((SPSS) IBM Corporation) for survey questions that could be converted into an ordinal data set.

\section{RESULTS}

One hundred and five (105) participants were recruited following full description of the study and inclusion criteria. Of the 105 participants recruited, 79 met inclusion criteria. No child surveys were returned. According to caregiver report of which type of lower limb orthotic the user wore, $48.1 \%$ wore an Ankle Foot Orthotic (AFO), 13.9\% wore a hinged Ankle Foot Orthotic, 13.9\% wore something other, $10.1 \%$ wore a Supra Malleolar Orthotic (SMO), 5.1\% used a Hip Knee Ankle Foot Orthotic (HKAFO), 5.1\% used the Dynamic Ankle Foot Orthotic (DAFO), and 3.8\% used the Knee Ankle Foot Orthotic (KAFO). In total, according to caregiver report, 36 individuals, (50\%) reported that the user wore their device 4-6 hours per day, 24 individuals (33.35\%) wore their devices 1-4 hours per day, 8 individuals (11.1\%) wore their devices 30 minutes- 1 hour per day, and 4 individuals (5.6\%) were not wearing their device every day. In addition,

- $90 \%$ of participants reported that orthotics affect what type of shoe the user can wear

- $85.9 \%$ of participants reported that the user requires larger shoes to accommodate for the size of the orthotic

- $82 \%$ reported that it is difficult to shop for shoes that accommodate orthotics

- $68.3 \%$ of participants reported that the user would be more likely to wear their orthotics if they had shoes that took less time to put on

- $67 \%$ reported there are times the user does not wear the orthotics due to the individual wanting to wear shoes that do not accommodate the orthotic

- $61 \%$ reported that the user would be more likely to wear their orthotics if they had more options of shoes to wear

- $59 \%$ reported that the user would be more likely to wear their orthotics if they had shoes that were more attractive 


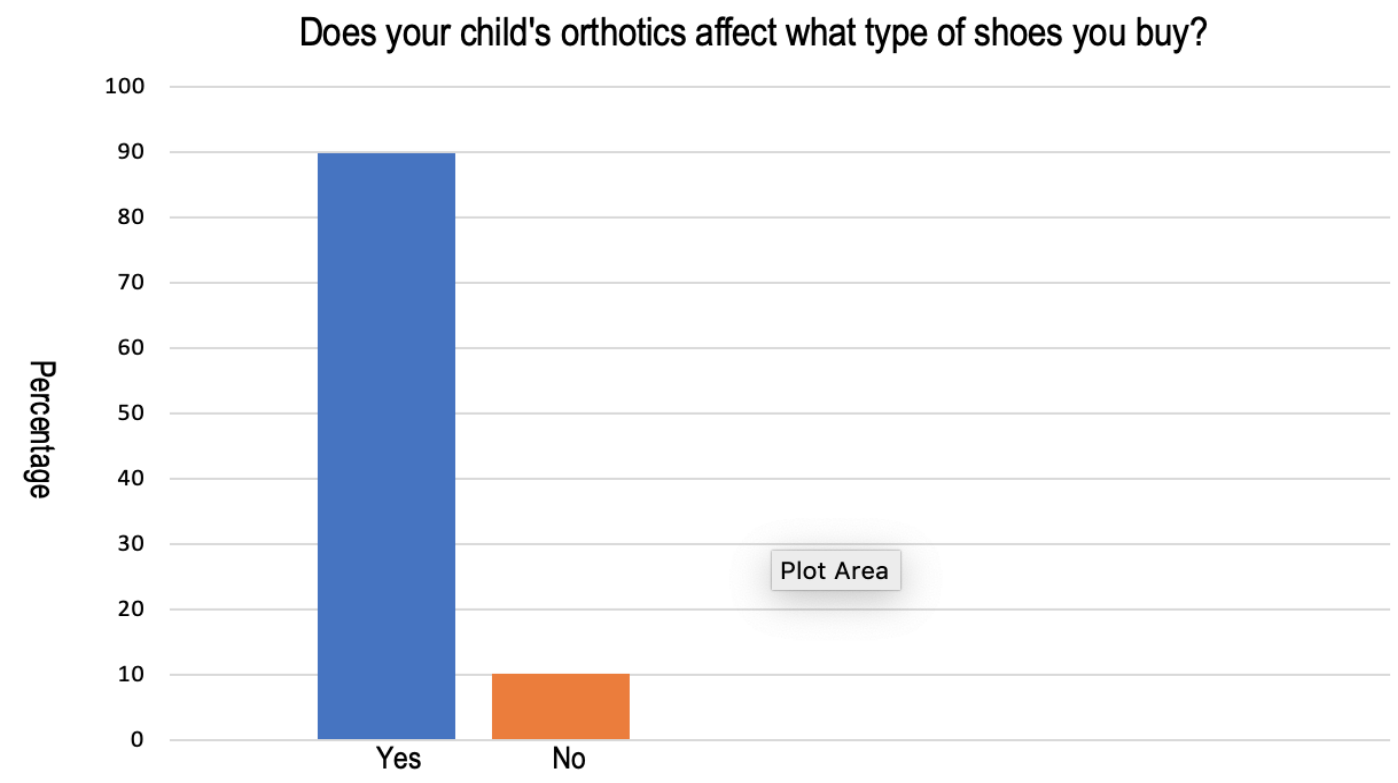

Figure 1. Percentage of responses to the question: "Does your child's orthotics affect what type of shoes you buy?".

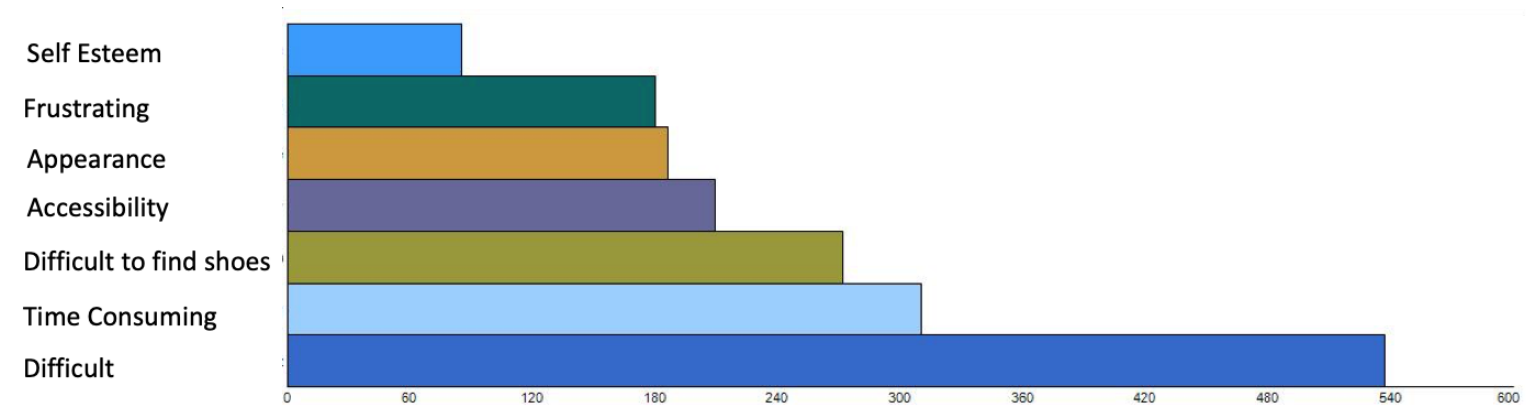

Figure 2. Bar graph produced by Nvivo 10 representing word frequency of the 7 identified themes from the answers to the question, "Describe your experiences with putting on and taking off your child's orthotic(s) and shoes."

\section{Theme 1: Difficult}

Of the caregivers who answered the statement "describe your experiences with putting on and taking off your child's orthotic(s) and shoes," there were 540 words that described the process as being "difficult" (or a synonym) (Figure 2). A few quotes from caregivers that reflect the difficulty of the process include:

- "Putting shoes on is very difficult as shoes do not have enough give."

- "Shoes are so hard to put on that sometimes we just call [it] quits with the shoes."

- "We tried on 15+ pairs of shoes before I gave up."

- "Special knee-high socks on first, then the insert fix chip, place outer shell on strap the three straps then repeat with other foot. Take shoes super loosened then squish and push his foot into his extra wide new balance shoes then have him stand to get the heel in then tighten and tie then repeat with other foot. It is a pain..."

\section{Theme 2: Time Consuming}

Of the caregivers who answered the statement "describe your experiences with putting on and taking off your child's orthotic(s) and shoes,", there were over 300 words that described the process as being "time consuming" (or a synonym) (Figure 2). A few quotes from caregivers that reflect the time-consuming nature of the process include: 
- $\quad$ "Time-consuming, counter-productive to maximizing boot wearing, and stressful for child (squirming, crying) and parent (frustrating, guilt, sadness) Boots often slip off on their own, causing blisters and increased instances of reapplying boots. Makes for a very unpleasant day."

- "It is time consuming. Wearing them is cumbersome."

\section{Theme 3: Difficult to find shoes}

Amongst the qualitative questions in the survey, many participants expressed how difficult it is to find shoes that accommodate the user's orthotics:

- "My child wears [orthotics] anyways as she is more dependent on her wheelchair without them. She frequently complains of the shoe styles not available to her because of her braces, though."

- "Limited shoe wear is the most difficult aspect of my daughter wearing orthotics."

- "We would love to have more shoe options! She is only able to wear athletic shoes. It would be amazing to be able to wear dress shoes or boots."

- " "It's VERY hard to find shoes that come in wide or extra wide that have closures my son can use independently."

- "We struggle to find a shoe that will fit and isn't ridiculously large."

As some users wear an orthotic on only one foot, two different sized shoes may be needed. The following quote explains how difficult it can be to find two different sizes of the same shoe:

- "Most stores won't let you purchase 2 different sized shoes without paying for 2 pairs."

\section{Theme 4: Accessibility}

- "He can't wear most style of shoes because they are not wide enough. It makes him really upset."

- "The extra weight of shoes big enough to wear over braces caused great delays in getting used to the braces and learning to walk. In toddler sizes, even a little extra weight and size are a big difference."

- "No shoes fit my child's braces unless custom made."

- "Not all stores have shoes that fit, most are expensive never satisfied at the final results."

- "Most stores do not carry shoes wide enough. When we order wide shows they don't always fit well."

- "Shoes should be accessible to all children."

Theme 5: Appearance

The appearance of footwear is a very important aspect for some individuals and caregivers. The following quotes are answers to various open-ended questions throughout the survey and address the concept of appearance:

- "The custom shoes are hideous."

- "He loves light up shoes and slip-ons. Neither are really an option with braces."

- "Sometimes the shoes don't fit because of the AFOs. Cute dressy shoes are hard to find."

- $\quad$ "Shoes are difficult to find, and my daughter is very "girly" wanting sparkles so refuses to wear many of the shoes that will work. When we do find some, they are difficult to get on and she needs help to do it, often limiting the amount of time she actually wears them."

- "He wants the shoes that light up."

\section{Theme 6: Frustrating}

In response to the statement, "describe your experiences with putting on and taking off your child's orthotic(s) and shoes", caregivers responded with the following answers that expressed frustration with the process:

- "The brace has to be put on perfectly or blisters will be caused."

- "It's not a fun activity."

- "massively frustrating." 


\section{Theme 7: Self-esteem}

In response to the question, "Does your child's orthotics affect what type of shoes your child is able to wear?", caregivers responded with the following answers that expressed how their child experienced decreased self-esteem due to orthotics/shoe options:

- "My daughter is not able to wear shoes that most teens wear."

- "She is getting to the age where she wants a say in her clothing, but shoe choices are limited."

When asked the question "describe your experiences with putting on and taking off your child's orthotic(s) and shoes", one caregiver stated:

- "It is very discouraging for my daughter to see her friends put their shoes on independently, but she cannot.

\section{DISCUSSION}

Regarding the perceptions of orthotics and footwear, the results demonstrate caregiver frustration with the process of donning/doffing orthotics and footwear as well as with shopping for footwear to accommodate for the size of orthotics. With this information, it is important for professionals involved in the design phase of footwear to consider the client's perceptions to create a product that is meeting the needs of the population. Current research suggests that "achieving the clinical benefits of footwear is not just about getting the footwear designed and fitted to meet the clinical needs [of the patient], but it also requires that the patient wears the shoes. This means meeting the non-clinical needs or criteria of patients." 5 Some examples of clinical benefits of footwear are extra wide shoes, extra deep shoes, or shoes of differing heights. The aspect that is often not considered during the design phase of footwear production is the non-clinical needs of patients; this includes aspects such as appearance, ease of use, and cost. With a client-centered approach to the design of footwear to accommodate orthotics, the needs of the population will be met and therefore increase participation in meaningful activities which contributes to one's physical, psychological, and social wellbeing. ${ }^{9}$ While research is limited, current research suggests that footwear is strongly connected to an individual's body image and self-esteem and thus, their mood, depression, and quality of life. ${ }^{6,5}$ Many caregivers that participated in this study mentioned concepts relating to poor self-esteem due to the user being unable to wear the same shoes as their friends. With this information, it becomes apparent that it is important to take into consideration the needs, wants, and considerations of the clients and thus, further investigate patient perceptions of footwear and incorporate client choice into the design process.

Because orthotics are typically very large and wide, shopping for footwear has the potential to be very difficult. As $82 \%$ of caregivers reported that it is difficult to shop for shoes that accommodate orthotics, it is important for health care providers and manufacturers to advocate for this population and thus have products that meet the needs of our client's more readily available. Most participants reported that the user wears shoes that are 1.5 sizes larger than their true size in order to accommodate for their orthotic. This presents major problems for individuals who are able to ambulate as there is an increased risk for fall related injuries. It is important for designers to consider this when designing footwear as there may be alternative options to eliminate this problem. In relation to the style of footwear, many caregivers in this study reported that the users wear their orthotics regardless of the style of the shoe due to the orthotics being essential for function. This is consistent with research found by Anderson et al as the workers that participated in the study reported, "style was secondary to the function of the shoe." ${ }^{10} \mathrm{~A}$ little more than sixty eight percent $(68.3 \%)$ of caregivers from this study reported that their child would be more likely to wear their orthotics if they had shoes that took less time to put on. In a study about footwear and workers, the ease of donning and doffing footwear was also very important, as many participants described this as a requirement in the ideal footwear. ${ }^{9}$

It is important to note that the age of the orthotic user could have influenced answers given by caregivers as individuals who have been wearing orthotics for many years may not view the appearance of their shoes as important anymore or they may not require as much time to don/doff footwear due to level of experience. It is also likely that the type of orthotic that the individual wore influenced the results. For example, it is likely that those who reported wearing SMO (supra malleolar) orthotics, may not have as hard of a time finding shoes to accommodate the orthotics due to the orthotics not being as large and wide as other types. In addition, compliance with specific types of orthotics varies greatly depending on the type of orthotic that is prescribed.

\section{Limitations}

Possible limitations to this study include that the method of gathering data was through surveys which is subject to potential response bias. This includes "respondents being unable to recall information accurately, interpreting the meaning of a question differently than the meaning intended by the researcher, or response choices that do not accurately express respondents' experiences or opinions." ${ }^{11}$ This study was intended to gain the perspectives of orthotic users directly from those that use orthotics, however, no surveys were returned from individuals who met the inclusion criteria for those who wore orthotics. Surveys from 
individuals who wear lower limb orthotics may have not been returned due to the cognitive ability of the individual or the wording of the survey was too complex. The surveys that were answered were not validated and in turn, no psychometric properties have been established. Another limitation is that through Facebook support groups, only caregivers were able to provide responses to the survey which limits the perspectives gathered. It is also a limitation that data regarding participants characteristics/demographics such as age, gender, and diagnosis were not collected.

\section{CONCLUSIONS}

It is important to explore the perceptions of individuals who use lower limb orthotics and their caregivers to inform the design of products, in particular, footwear. Health care providers who prescribe footwear and orthotics should consider the needs of the population to facilitate physical, emotional, and social health. The results of this study are of clinical interest and suggests that this is an important area to research to improve the quality of life of individuals who use orthotics. It is essential to inform the development and production of footwear to promote the adherence to the prescription of orthotic use. By exploring and understanding the needs of individuals who use lower limb orthotics, designers and manufacturers will be able to create footwear that meets both the personal needs of the population as well as allow the orthotic to function as medically necessary. Future studies should seek to gain the perspectives of individuals who use lower limb orthotics in order to further research this population's specific needs.

\section{References}

1. U.S. Census Bureau. (2017). American Community Survey 1-Year Estimates

2. American Occupational Therapy Association (AOTA). (2010). Specialized knowledge and skills in technology and environmental interventions for occupational therapy practice Am J Occup Ther, 64, S44-S56. doi:10.5014/ajot.2010.64S44

3. Johnston, P., Currie, L. M., Drynan, D., Stainton, T., \& Jongbloed, L. (2014). Getting it "right": How collaborative relationships between people with disabilities and professionals can lead to the acquisition of needed assistive technology. Disabil Rehabil Assis Technol,9(5), 421-431. doi:10.3109/17483107.2014.900574

4. Pressman, S. D., Matthews, K. A., Cohen, S., Martire, L. M., Scheier, M., Baum, A., \& Schulz, R. (2009). Association of enjoyable leisure activities with psychological and physical well-being. Psychosom Med, 71(7), 725-732. doi:10.1097/PSY.0b013e3181ad7978

5. Williams, A. E., \& Nester, C. J. (2006). Patient perceptions of stock footwear design features. Prosthet Orthot Int 30(1), 6171. doi:10.1080/03093640600574425

6. Polliack, A. A., Elliot, S., Caves, C., Mcneal, D. R., \& Landsberger, S. E. (2001). Lower Extremity Orthoses for Children with Myelomeningocele: User and Orthotist Perspectives. J Prosthet Orthot13(4), 123-129. doi:10.1097/00008526-20011200000012

7. Corcoran, M.A. (2006). Using Mixed Methods Designs to Study Therapy and Its Outcomes. In Kielhofner, G., Research In Occupational Therapy: Methods of Inquiry for Enhancing Practice. (pp. 411). Philadelphia, PA: F.A. Davis Company.

8. Dillaway, H., Lysack, C., Luborsky, M.R. (2006). Qualitative Approaches to Interpreting and Reporting Data. In Kielhofner, G., Research In Occupational Therapy: Methods of Inquiry for Enhancing Practice. (pp. 373). Philadelphia, PA: F.A. Davis Company.

9. Law, M. (2002). Participation in the occupations of everyday life. Am J Occup Ther, 56, 640-649. doi:10.5014/ajot.56.6.640

10. Anderson, J., Williams, A. E., \& Nester, C. (2017). An explorative qualitative study to determine the footwear needs of workers in standing environments. J Foot Ankle Res, 10(1). doi:10.1186/s13047-017-0223-4

11. Forsyth, K. \& Kviz, F.J. (2006). Survey Research Design. In Kielhofner, G., Research In Occupational Therapy: Methods of Inquiry for Enhancing Practice. (pp. 91-109). Philadelphia, PA: F.A. Davis Company. 